\title{
Nonlinear Blind Source Separation for EEG Signal Pre-processing in Brain-Computer Interface System for Epilepsy
}

\author{
D. A. Torse \\ Gogte Institute of Technology, \\ Belgaum, Karnataka
}

\author{
R. R. Maggavi \\ KLE's College of Engineering \\ and Technology, \\ Belgaum, Karnataka
}

\author{
S. A. Pujari \\ Jaipur Engineering College, \\ Kukas, Jaipur, Rajasthan
}

\begin{abstract}
Epilepsy is one of the most common neurological disorders characterized by transient and unexpected electrical disturbance of the brain. The electroencephalogram (EEG) potentials represent the combined effect of potentials from a fairly wide region of the scalp. Mixing some underlying components of brain activity apparently generates these potentials. The aim of the present study is to separate the original components of brain activity waveforms from their linear mixture. The probability distributions and mixing coefficients knowledge is not considered. This is called the problem of "Nonlinear Blind Source Separation" (NBSS). It consists of the recovery of unobservable original independent components from several mixed components covered by mixed sources. The current study used recently developed source separation method known as "Independent Component Analysis" (ICA) technique for solving blind EEG source separation problem. The proposed ICA NBSS model has been implemented using the Matlab version 7.7. The measured real EEG data signals obtained from epileptic states. The results of the present work show the good performance of the proposed model in separating the mixed signals.
\end{abstract}

\section{Keywords}

Electroencephalogram, Principal component analysis, Nonlinear Blind Source Separation, EEG based BCI.

\section{INTRODUCTION}

Epilepsy, a chronic disorder of the nervous system, is characterized by the abnormal synchronized firing of a large number of neurons. This large synchronized event is known as a seizure, paroxysmal discharge, or ictal event. Abnormal activity is almost always observed between seizures and is known as interictal activity. These interictal events are short in duration but occur more frequently than ictal events. Epilepsy has a variety of causes. The genetic component, head trauma, infection, stroke, hypoxia, dysplasia, and various chemical imbalances in the brain can also cause epilepsy [1].

The EEG signals result from the activity of neurons various significant distances away from the electrodes place on the scalp. Each electrode is a summation of the electrical neural activity of a large number of individual neurons in the surrounding area; therefore because of the distance between the skull and brain, and their different resistivities,

Manuscript received June , 2012; revised June , 2012; accepted datorse@git.edu,raghu_maggavi@indiatimes.com, sapujari@rediffmail.com electroencephalographic data collected from any point on the human scalp includes a mixture of activity generated within a large brain area [2].

The importance of signal processing and analysis of EEG waveforms based on computer encouraged the scientists in their work [3]. The event related potential (ERP) data shows that the algorithm can isolate EEG artifacts, since the result proved that this algorithm is able to decompose EEG artifacts such as line noise, eye blinks, muscle activity, and cardiac noise [4]. The use of the extended ICA algorithm to investigate task related human brain activity in fMRI data has been presented in [5]. A fixed point algorithm, which utilizes extraction sleep spindles from the (EEG) and channel isolation of these sleep spindles from a multi-channel electroencephalograph has been proposed in [6]. A novel model for classification of EEG data based on ICA as a feature extraction technique, and on evolving fuzzy neural networks as a classification modeling technique has been presented by [7]. The study in [8] demonstrated that ICA can dramatically improve the classification of (EEG) from various conditions. The major objective of this paper is to study and design methods to analyze the EEG signals. Here we examine the relation between EEG signals and the Nonlinear Blind Source Separation (BSS) and study the ICA technique and its relation with NBSS in detail. A method implemented here is an algorithm based on ICA to pre-process the underlying signal of the epileptic EEG signals. These extracted EEG signals with the help of neurologists and psychologists in diagnosing and treating various neurological disorders.

\section{EEG BASED BCI SYSTEM}

Approximately $1 \%$ population (3 million) in US is suffering from epilepsy [9]. Epilepsy is the second most common brain disorder after Stroke. Severe mental disorders that include stroke, epilepsy, bipolar disorder, organic psychosis and major depression affect nearly 20 per 1000 population in India [10]. More than half remain without treatment due to lack of diagnosis and treatment systems. The data reveals that there is only one psychiatrist available for every 3 lacks population in India. The psychiatrist/population ratio is under one for every million in rural areas that account for $70 \%$ of country's population [10].

The origins of Brain-Computer Interface (BCI) for the treatment of clinical disorders can be directly traced to the first systematic demonstration of the electroencephalogram, or EEG operant conditioning in general [11]. In the context of sleep research, Sterman and associates [11] conducted a series of studies investigating a spectral peak around $12-14 \mathrm{~Hz}$, 
and was referred to as the "sensorimotor rhythm" (SMR). BCI training can be used to gain control over the SMR activity. BCI researchers believe and have produced experimental evidence to back up their claim that this feedback enables the user to learn the regulation of their own SMR.

$\mathrm{BCI}$ is a direct training of brain function, by which the brain learns to function more efficiently. With the brain in action from moment to moment $\mathrm{BCI}$ ensures that information flows back to the person and rewards the brain for changing its own activity to more appropriate patterns. This is a gradual learning process. It applies to any aspect of brain function that is measured. The BCI self-regulation training allows the central nervous system to function better [12].

The system introduced above is a non-invasive method for treating neurological disorders such as epilepsy, Attention Deficit Hyperactivity Disorder (ADHD) and autism.

Figure 1 shows the general model of an EEG based BCI system. The figure depicts a generic BCI system in which a person controls a device in an operating environment [13] (e.g., a video game on computer screen) through a series of functional components.

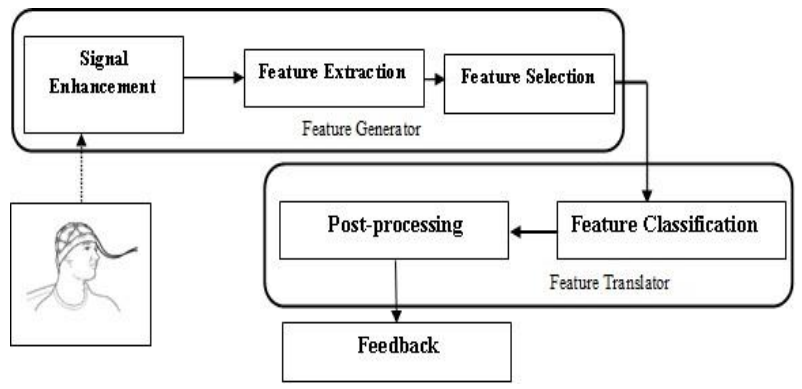

Fig 1: General model of an EEG based BCI system.

In this context, the user's brain activity is used to generate the control signals that operate the BCI system. The user monitors the state of the device to determine the result of his/her control efforts. The electrodes placed on the scalp of the user record the brain signal from the neural activity within the brain, and convert this brain activity to electrical signals. The 'feature generator' block transforms the resultant signals into feature values that correspond to the underlying neurological mechanism employed by the user for control. For example, if the user is to control the power of his/her theta $(4-8 \mathrm{~Hz})$ and beta $(13-30 \mathrm{~Hz})$ rhythms, the feature generator would continually generate features relating to the power spectral estimates of the user's theta and beta rhythms. The feature generator generally consists of three components i.e. 'signal enhancement', 'feature extraction' and 'feature selection'. The term 'signal enhancement' refers to the pre-processing stage.

A 'feature selection' component is sometimes added to the BCI system after the 'feature extraction' stage. The aim of this component is to reduce the number of features and/or channels used so that very high dimensional and noisy data can be excluded. Ideally, the features that are meaningful or useful in the classification stage are identified and chosen. The 'feature translator' translates the features into logical control signals, such as a two-state discrete output. As shown in figure 1 , the feature translation block may consist of two components: 'feature classification' and 'post-processing'. Finally the control signal generated is used as a feedback signal in the form of audio and video.

\section{ORGANIZATION OF BRAIN}

An EEG signal is a measurement of currents that flow during synaptic excitations of the dendrites of many pyramidal neurons in the cerebral cortex. When neurons are activated, the synaptic currents are produced within the dendrites. This current generates a magnetic field measurable by electromyogram (EMG) machines and a secondary electrical field over the scalp measurable by EEG systems. Differences of electrical potentials are caused by summed postsynaptic graded potentials from pyramidal cells that create electrical dipoles between the soma (body of a neuron) and apical dendrites, which branch from neurons as shown in figure 2 .

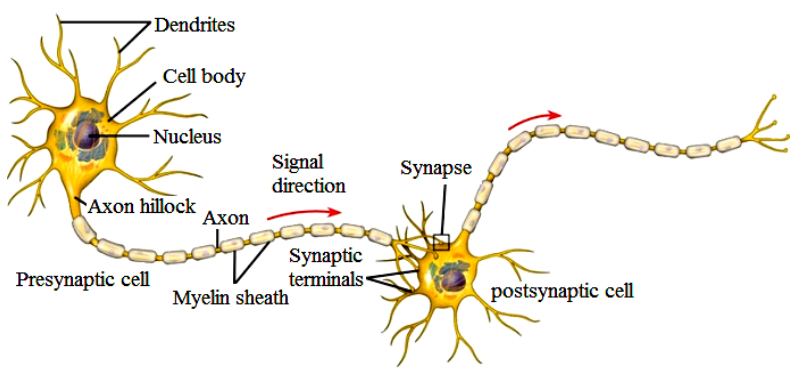

Fig 2: The structure of a typical neuron [18].

The current in the brain is generated mostly by pumping the positive ions of sodium, $\mathrm{Na}+$, potassium, $\mathrm{K}+$, calcium, $\mathrm{Ca}++$, and the negative ion of chlorine, $\mathrm{Cl}-$, through the neuron membranes in the direction ruled by the membrane potential [14]. The human head consists of different thin layers including the scalp, skull, brain as depicted in figure 3 . Approximately one hundred times more than the soft tissue the signal attenuation takes place due to skull. Most of the noise generated in the brain is due to internal noise or system noise which is external. Only large populations of active neurons can generate enough potential to be recordable using the scalp electrodes. At birth when the central nervous system becomes complete and functional, approximately $10^{11}$ neurons are developed [15]. This makes an average of $10^{4}$ neurons per cubic mm. Neurons are interconnected into neural nets through synapses. The number of synapses per neuron increases with age, whereas the number of neurons decreases with age. From an anatomical point of view the brain may be divided into three parts: the cerebrum, cerebellum, and brain stem as shown in figure 4 .

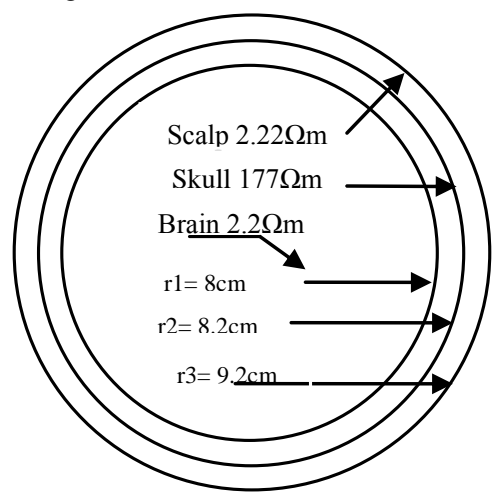

Fig 3: The three main layers of the brain including their approximate resistivities and thicknesses $(\Omega=\mathrm{ohm})$ [18].

The cerebrum consists of both left and right lobes of the brain with highly convoluted surface layers called the cerebral cortex. The cerebrum includes the regions for movement initiation, conscious awareness of sensation, complex 
analysis, and expression of emotions and behavior. The cerebellum coordinates voluntary movements of muscles and maintains balance. The brain stem controls involuntary functions such as respiration, heart regulation, biorhythms, and neuro-hormone and hormone sections [16]. This indicates that the study of EEGs shows the way for diagnosis and treatment of neurological disorders such as epilepsy. The acquired EEG signals from a human can be used for investigation of the clinical problems $[16,17]$.

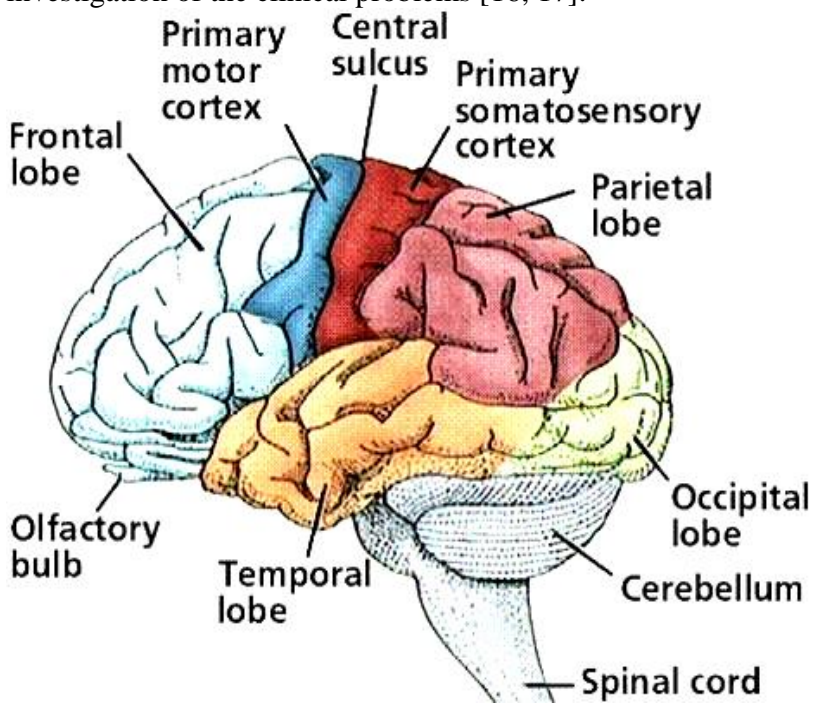

Fig 4: Diagrammatic representation of the major parts of the brain[18].

The rich potential for EEG analysis and motivates the need for advanced signal processing techniques to assist the neurologists in their diagnosis and treatment [18].

\subsection{Brain Wave Types}

Many brain disorders are diagnosed by visual inspection of EEG signals. The clinical experts in the field are familiar with expression of brain rhythms in the EEG signals. In healthy adults, the amplitudes and frequencies of such signals change from one state of a human to another, such as wakefulness and sleep. The age is also a factor with which the characteristics of brain wave changes. There are five major brain waves distinguished by their different frequency ranges. These frequency bands from low to high frequencies respectively are called alpha $(\alpha)$, theta $(\theta)$, beta $(\beta)$, delta $(\delta)$, and gamma $(\gamma)$.

The alpha and beta waves were introduced by Berger in 1929 . The term 'gamma' was used to indicate $30 \mathrm{~Hz}$ above waves. Walter introduced delta waves in 1936 to assign all frequencies below the alpha range. Also theta waves having frequencies within the range of $4-7.5 \mathrm{~Hz}$ were introduced by Walter [19]. The range of delta waves lies within the 0.5-4 $\mathrm{Hz}$. These waves are primarily associated with deep sleep and related to displeasure, pleasure, and drowsiness.

The signals from large muscles of the neck and jaw can very easily mix with real delta waves as the muscles are near the surface of the skin and produce large signals. The concerned signal originates from deep within the brain and is severely attenuated in passing through the skull. The application of simple signal analysis methods to the EEG, it is very easy to see when the response is caused by excessive movement

Theta waves lie within the range of $4-7.5 \mathrm{~Hz}$. The term theta might be chosen to allude to its presumed thalamic origin. Theta waves appear as consciousness slips towards drowsiness. Theta waves have been associated with access to unconscious material, creative inspiration and deep meditation. The theta wave plays an important role in infancy and childhood. Larger contingents of theta wave activity in the waking adult are abnormal and are caused by various pathological problems. The changes in the rhythm of theta waves are examined for maturational and emotional studies [20].

Alpha waves appear in the posterior half of the head and are usually found over the occipital region of the brain. They can be detected in all parts of posterior lobes of the brain. The alpha waves frequency lies within the range of $8-13 \mathrm{~Hz}$. A relaxed awareness without any attention or concentration is indicated by these waves. In most cases alpha waves are produced with eyes closed. A waiting pattern produced by the visual regions of the brain is also called alpha wave. It is reduced or eliminated by opening the eyes, by hearing unfamiliar sounds, by anxiety, or mental concentration or attention. To understand the origin and physiological significance of alpha waves yet more research has to be undertaken [21].

A beta wave is the electrical activity of the brain varying within the range of $14-26 \mathrm{~Hz}$ (though in some literature no upper bound is given). A beta wave is the usual waking rhythm of the brain associated with active thinking, active attention, focus on the outside world, or solving concrete problems, and is found in normal adults.

A high-level beta wave may be acquired when a human is in a panic state. Rhythmical beta activity is encountered chiefly over the frontal and central regions. Importantly, a central beta rhythm is related to the rolandic mu rhythm and can be blocked by motor activity or tactile stimulation. The amplitude of beta rhythm is normally under $30 \mu \mathrm{V}$. Similar to the mu rhythm, the beta wave may also be enhanced because of a bone defect and also around tumoural regions.

The gamma range corresponds to frequencies above $30 \mathrm{~Hz}$. The amplitudes of these rhythms are very low and their occurrence is rare. Some brain disorders are detected by use of these waves. The gamma wave band has also been proved to be a good indication of event-related synchronization (ERS) of the brain [22].

The sensorimotor rhythm i.e. SMR waves play important role in detecting epileptic states in few cases. [23].

BETA $13-30 \mathrm{~Hz}$
DELTA $0.5-4 \mathrm{~Hz}$
Figure 5 shows the typical normal brain rhythms with their
usual amplitude levels. In general, the EEG signals are the
projection of neural activities that are attenuated by the scalp.
Cartographic discharges show amplitudes of $0.5-1.5 \mathrm{mV}$ and
up to several millivolts for spikes. However, on the scalp the
amplitudes commonly lie within 10-100 $\mu \mathrm{V}$. It is often


difficult to understand and detect the brain rhythms from the scalp EEGs, even with trained eyes. The application of advanced signal processing tools allows separation and analysis of the desired waveforms from within the EEGs. Therefore, a definition of foreground and background EEG is very application specific and depends only on the abnormalities. Next we study the method of the recording of EEG data for the proposed scheme.

\subsection{EEG Recording Mode}

The International Federation of Societies for Electroencephalography and Clinical Neurophysiology has recommended the conventional electrode setting (also called 10-20) for 21 electrodes (excluding the earlobe electrodes), as depicted in Figure 6 [24].

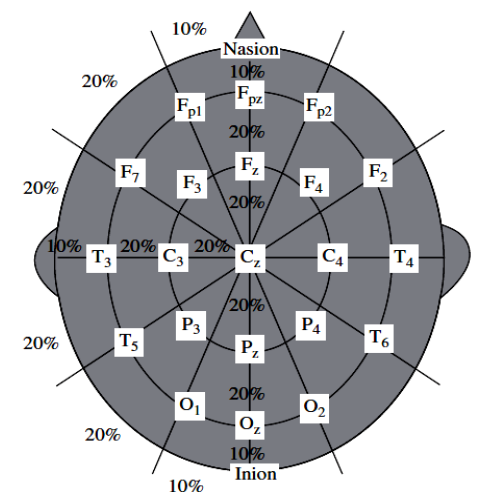

Fig 6: Conventional 10-20 EEG electrode positions for the placement of 21 electrodes [18].

The earlobe electrodes called $\mathrm{A}_{1}$ and $\mathrm{A}_{2}$, connected respectively to the left and right earlobes. The Maudsley electrode positioning system which is the modification of conventional 10-20 system captures better signals from epileptic foci in epileptic seizure recordings. In this system the outer electrodes are slightly lowered to enable better capturing of the required signals. A more extensive coverage of the lower part of the cerebral convexity is obtained in this method and thus increases the sensitivity for the recording from basal subtemporal structures [25]. Other deviations from the international 10-20 system as used by researchers are found in References [26] and [27]. In EEG based BCI often a small number of electrodes around the movement-related regions are selected and used from the 10-20 setting system.

\section{INDEPENDENT COMPONENT \\ ANALYSIS}

The concept of Independent Component Analysis (ICA) lies in the fact that the signals may be decomposed into their constituent independent components. In places where the combined source signals can be assumed independent from each other this concept plays a crucial role in separation and denoising the signals. A measure of independency may easily be described to evaluate the independence of the decomposed components. Generally, considering the multichannel signal as $y(n)$ and the constituent signal components as $y_{i}(n)$, the $y_{i}(n)$ are independent if,

$P_{y}(y(n))=\prod_{i=1}^{m} P_{y}\left(y_{i}(n)\right) \quad \forall_{n}$ where $P(Y)$ is the joint probability distribution, $p_{y}\left(y_{i}(n)\right)$ are the marginal distributions and $m$ is the number of independent components.

An important application of ICA is in blind source separation (BSS). BSS is an approach to estimate and recover the independent source signals using only the information of their mixtures observed at the recording channels. Due to its variety of applications BSS has attracted much attention recently. BSS of acoustic signals is often referred to as the "cocktail party problem' [28], which mean separation of individual sounds from a number of recordings in an uncontrolled environment such as a cocktail party. Figure 7 illustrates the BSS concept. As expected, ICA can be useful if the original sources are independent, i.e.

$P_{(s(n))}=\prod_{i=1}^{m} P_{i}\left(S_{i}(n)\right)$

A perfect separation of the signals requires taking into account the structure of the mixing process. In a real-life application, however, this process is unknown, but some assumptions may be made about the source statistics.

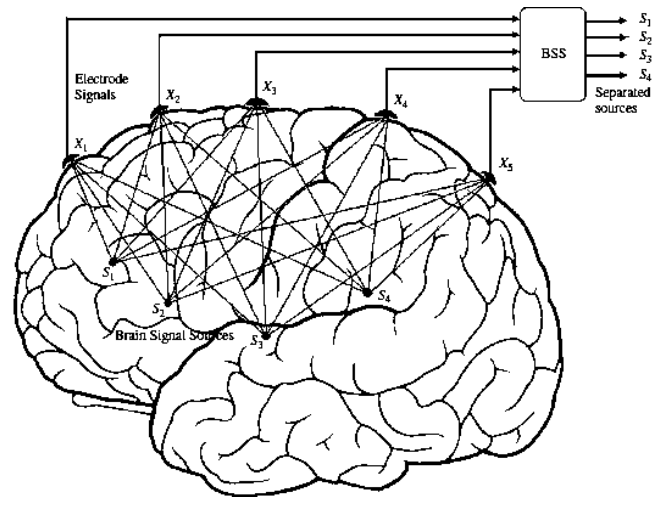

Fig 7: BSS concept; mixing and blind separation of the EEG signals [18].

The need to select the BSS algorithms is that it does not make realistic assumptions about the environment in order to make the problem more tractable. There are typically three assumptions about the mixing medium. The most simple but widely used case is the instantaneous case, where the source signals arrive at the sensors at the same time. This has been considered for separation of biological signals such as the EEG, where the signals have narrow bandwidths and the sampling frequency is normally low. The BSS model in this case can be easily formulated as,

$x(n)=H_{S}(n)+v(n)$

where $m \times n s(n), n_{e} \times 1 x(n)$ and $n_{e} \times 1 v(n)$ denote

respectively the vectors of source signals, observed signals, and noise at discrete time $n . H$ is the mixing matrix of size $n_{e}$ $\times \mathrm{m}$. The separation is performed by means of a separating $m$ $\times n_{e}$ matrix, $W$, which uses only the information about $x(n)$ to reconstruct the original source signals (or the independent components) as In the context of EEG signal processing ne denotes the number of electrodes. The early approaches in instantaneous BSS started from the work by Herault and Jutten [29] in 1986. In their approach, they considered nonGaussian sources with similar number of independent sources and mixtures. They proposed a solution based on a recurrent artificial neural network for separation of the sources.

In the context of EEG analysis, although the number of signals mixed at the electrodes seems to be limited, the 
number of sources corresponding to the neurons firing at a time can be enormous. However, the objective here is to study a certain rhythm in the brain the problem transformation to the time-frequency domain or the space-time-frequency domain is necessary. In such domains the sources are considered disjoint and generally sparse. Also it is important to consider that in the brain neurons encode data in a sparse way if their firing pattern is characterized by a long period of inactivity $[30,31]$.

\subsection{Nonlinear BSS}

Consider the cases where the parameters of the mixing system change because of changes in the mixing environment or change in the statistics of the sources. For example, if the images of both sides of a semi-transparent paper are photocopied the results will be two mixtures of the original sources. However, since the minimum observable grey level is black (or zero) and the maximum is white (say 1), the sum of the grey levels cannot go 100 EEG Signal Processing beyond these limits. This represents a nonlinear mixing system. As another example, think of the joint sounds heard from surface electrodes from over the skin. The mixing medium involves acoustic parameters of the body tissues. However, the tissues are not rigid. In such cases, if the tissues vibrate due to the sound energy then the mixing system will be a nonlinear system. The mixing and unmixing can generally be modeled respectively as

$$
\begin{aligned}
& x(n)=f\left(A_{S}(n)+n(n)\right) \\
& y(t)=g(w(n))
\end{aligned}
$$

where $f($.$) and g($.$) represent respectively the nonlinearities$ in the mixing and unmixing processes. There have been some attempts to solve nonlinear BSS problems, especially for separation of image mixtures [32, 33]. In one attempt [32] the mixing system has been modeled as a radial basis function (RBF) neural network. The parameters of this network are then computed iteratively.

\section{PROPOSED ALGORITHM}

An EEG data from five scalps with electrodes placement according to the International 10-20 system has been selected for this study. Two EoG signals are selected. The selected subjects are diagnosed with epilepsy.

The ability of nonlinear BSS of ICA is demonstrated to decompose multichannel epileptic EEG data into temporally independent components. In this work, five asynchronous single-channel EEG data periods, 3-seconds (600-points) data periods are selected. The collection of EEG data is done from 20 samples with electrode placement as explained in subsection 3.2. The nonlinear BSS ICA enhancement technique is performed on 10 second EEG period using Matlab 7.7 software in the EEGLAB v9.0.8.6b. The neural learning approach is used for eliminating the artifacts from the EEG data. For separating the selected data samples the NBSS ICA technique is employed. Finally to estimate the ICA vectors a neural network technique is used. The algorithm used in this work is illustrated in figure 8 .

A method to apply ICA to multichannel EEG recordings and remove a wide variety of artifacts from EEG records by eliminating the artifact signals onto the scalp sensors is adopted. The results presented here show that NBSS ICA can effectively detect, separate and remove activity in EEG records from a wide variety of artifact signals. The present ICA model relate to the neural network approach for blind source separation. The input to this model is a file of the selected EEG data file as a column vector.

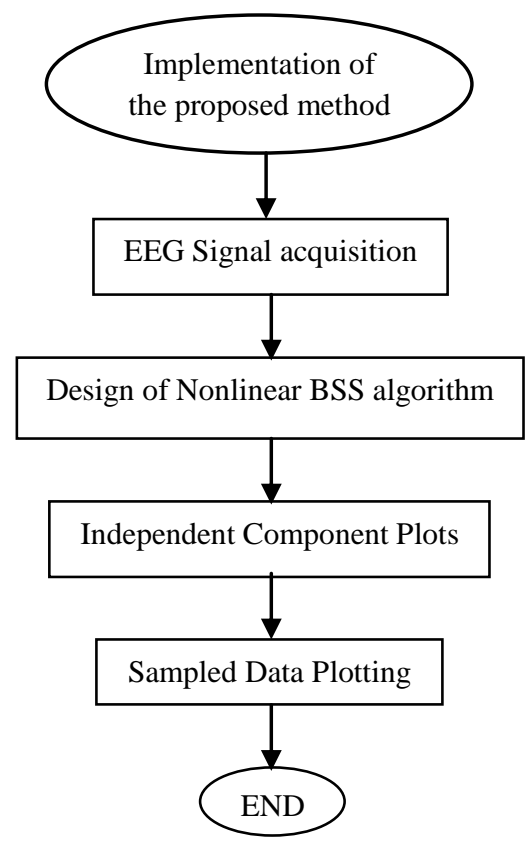

Fig 8: Flowchart of the algorithm.

\section{EXPERIMENTAL RESULTS}

Figure 9 demonstrates the ability of nonlinear BSS ICA to preprocess simulated EEG data. The proposed NBSS ICA enhanced EEG data and the correlation between each NBSS output waveform and its best correlated source waveform is shown in the figure.
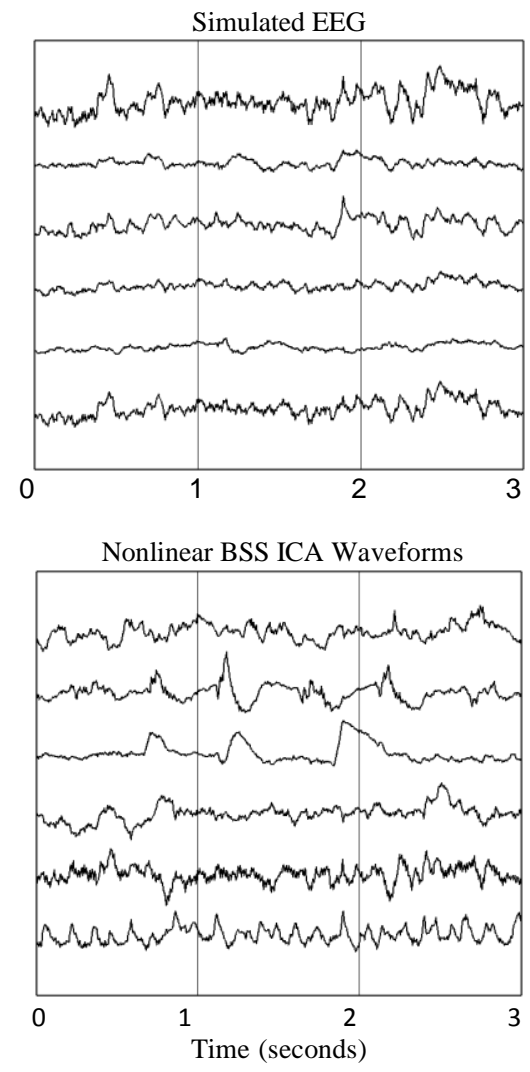


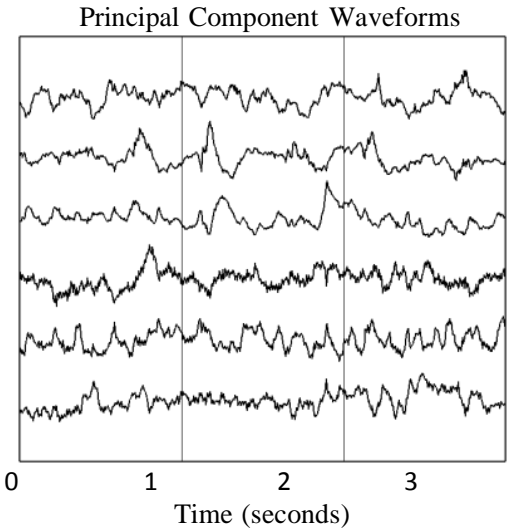

Fig 9: Simulated EEG, ICA, PCA separation

Figure 10 shows the derived components and the scalp topographies for the selected five components. The eye movement artifact between 1 and 3 second is separated to NICA components 12 and 15 . The muscle components are also represented. The corrected EEG signals obtained by eliminating the five selected components from the data are shown in figure 10 (b). The removal of these five artifact components by using nonlinear BSS by zeroing out the corresponding rows of the activation matrix projecting remaining components onto the scalp electrodes, the corrected EEG data is free from all artifact components.

Figure 11 depicts the five selected independent components. Figure 12 shows the Principal Component waveforms for the selected EEG data. The eye movement and muscle activity is corrected. It can be seen from the obtained results that the EEG recorded nearly becomes silent if the artifact comments are removed. When NBSS ICA effectively removes the eye movement artifact without affecting the activity of the EEG sources, it shows its effectiveness in the application to epileptic activity recorded.

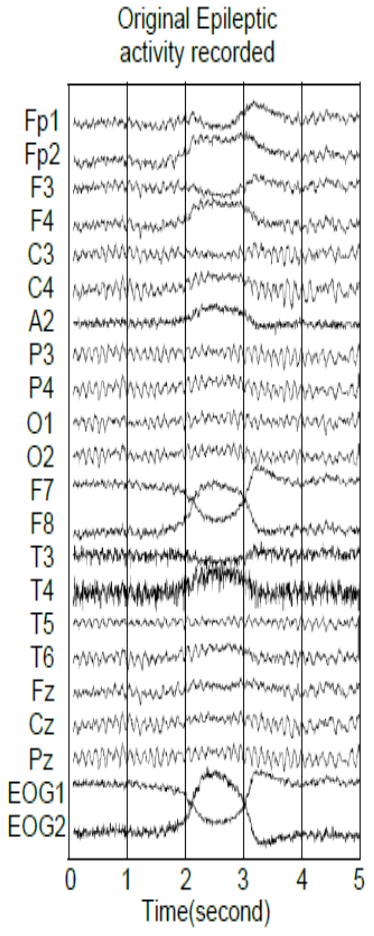

(a)

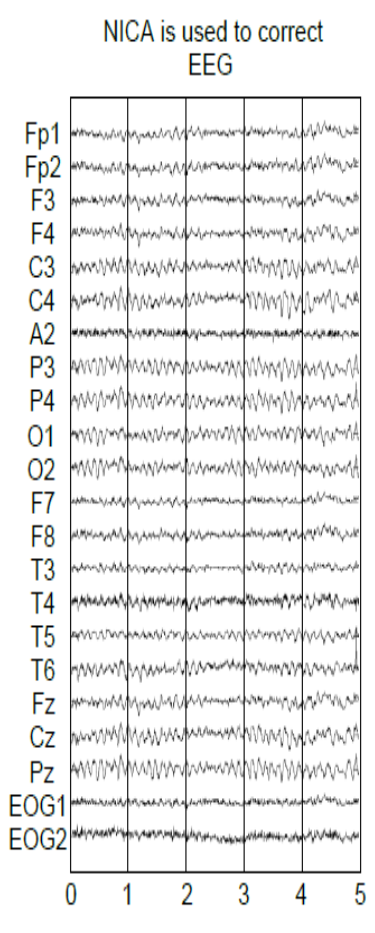

(b)
Fig 10: (a) Original epileptic activity, (b) NICA corrected EEG.

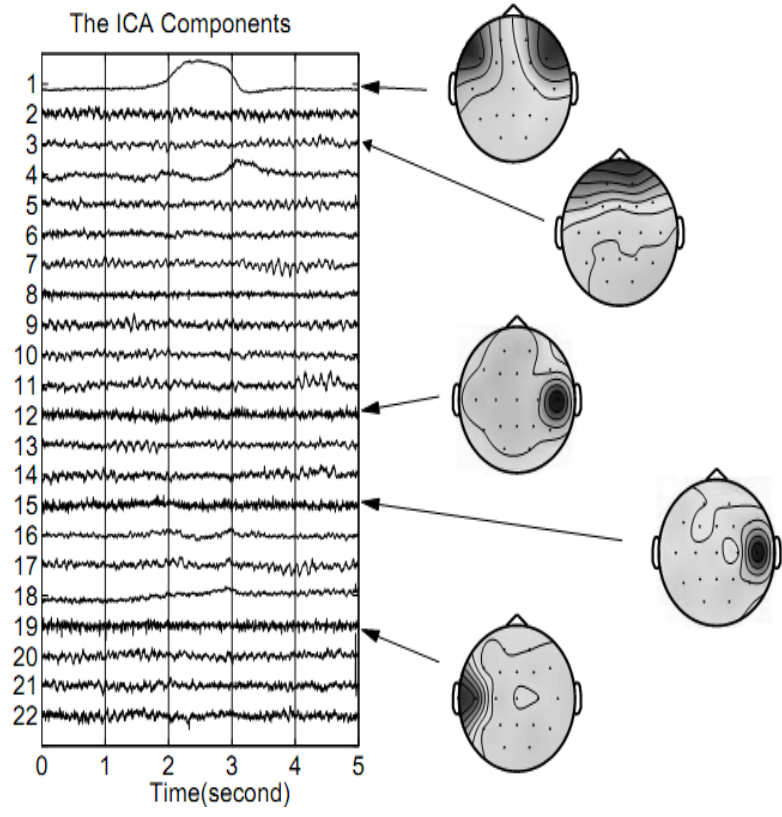

Fig 11: The five selected independent components.

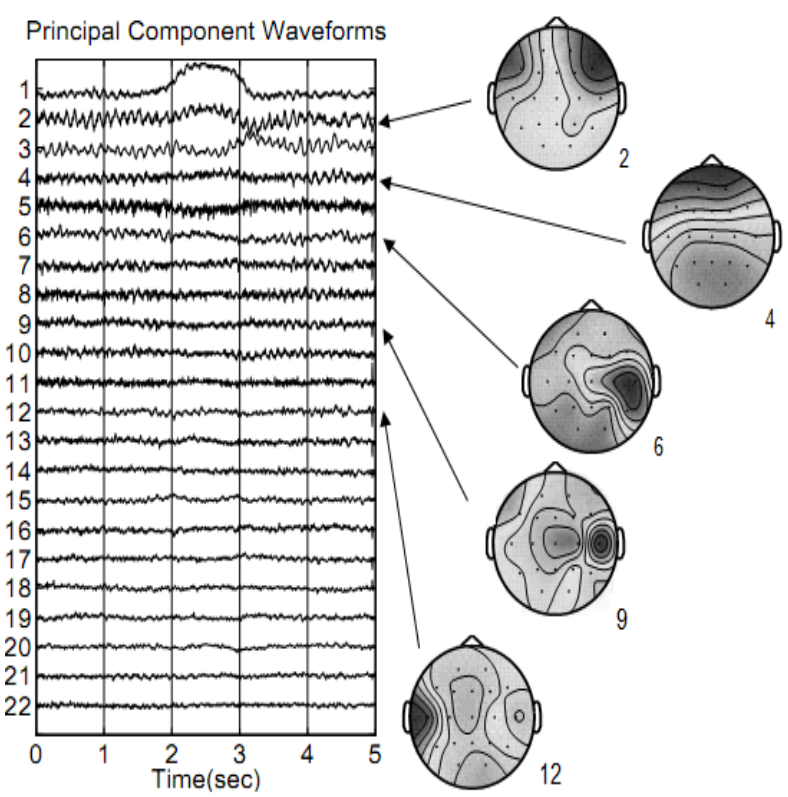

Fig 12: Selected five principal components.

\section{CONCLUSION}

The aim of this study is the design of a nonlinear BSS ICA neural network model, which can be used to analyze the complex epileptic EEG signals which are assumed to be blind signals. This technique is very useful in applications for, which there are observations. From EEG signal analysis, it is concluded that the epileptic recorded EEG signals are highly complex and non-Gaussian. Thus the conventional methods are limited to satisfy the present desire for extracting blind signals. This technique proved better pre-processing step for use in EEG based BCI systems. One way of gaining further insights on the EEG signals is to introduce nonlinear ICA

The present model of EEG analysis consists of two main stages: removing artifacts and separation. The removal of artifacts is successfully done by using PCA as a preprocessing stage. The PCA option is provided as a principled though imperfect way to make the training tractable for large 
numbers of channels only not for separation of independent components. While ICA is basically a way of separating and finding a special non-Gaussian data to perform a linear transform which makes the resulting variables as statistically independent for each other as possible. The Nonlinear BSS algorithm is used for separation. NBSS learning rule is used for ICA estimation. Indeed, almost any nonlinear function can be used in the learning rule. Thus one has a large freedom in the choice of the nonlinearity in the NPCA learning rule. This result is important because practically all other ICA procedures use a fixed nonlinearity or a limited number of them. From the extensive number of test performed using real EEG signal, the proposed model was found to be a very useful tool for doctor. This is due to the new signals, i.e. the independent components, which were presented.

The points planned for future work are to extend the present model to diagnose the brain disorders by constructing a huge information and database based on neurologist experiences, connect the ICA algorithms with the artificial intelligence techniques like fuzzy and genetic algorithms to extend their tasks to many other developed tasks such as classification and feature extraction of EEG waveforms and apply other signals processing and classification steps to develop a BCI system for managing epilepsy.

\section{REFERENCES}

[1] R. S. Fisher, "Epilepsy," in Pharmacological Management of Neurological and Psychiatric Disorders, S. J. Enna and J. T. Coyle, Eds: McGraw-Hill, 1998.

[2] Xiang-Yang, Z. and C. Yen-Wei, 1998. Signal separation by independent component analysis based on a genetic algorithms. Department of Electrical and Electronic Engineering, University of Okinawa,Japan.

[3] Makeig, S., T-P. Jung, and T.J. Sejnowski, 1996. Using Feedforward Neural Networks to Monitor Alertness from Changes in EEG Correlation and Coherence. In: Touretzky, D., M. Mozer and M . Hasselmo (Eds.), Advances in Neural Information Processing Systems. MIT Press, Cambridge, M A., 8: 931-937,

[4] T.P.Jung, S. Makeig and C. Humphries, 1998. Extended ICA removes artifacts from electroencephalographic recordings. Adv. Neural Info. Process. Sys., Cambridge, 10: 894-900.

[5] Mckeown, M. and S. Makeig, 1998. Blind separation of functional magnetic resonance imaging (fMRI) data, Hum. Brain Mapp., 6(5-6): 368-372.

[6] Barros, A.K., R. Rosipal, M. Girolami, G. Dorffner and N. Ohnishi, 2000. Extraction of Sleep-Spindles from the Electroencephalogram (EEG). In Artificial Neural Networks in Medicine and Biology (ANNIMAB-1), Göteborg, Sweden, Springer, pp: 125-130,

[7] Leichter C.S., A. Cichocki and N. Kasabov, 2001. Independent component analysis and evolving fuzzy neural networks for the classification of single trial EEG data. In: Proceeding 5th Biannu. Conf. Artificial Neural Network Expert System (ANNES 2001), pp: 100-105.

[8] Carl, S.E., C. Andrzej and K. Nik, 2000. Independent component analysis and evolving fuzzy neural networks for the classification of single trial EEG data. Department of Information Science, University of Otago, Dunedin, New Zealand.

[9] World Health Organization Epilepsy Fact Sheet, January 2011.
[10] Government of India, Department of Health and Family Welfare, Mental Health Programme, 2011.

[11] M. Barry Sterman \& Tobias Egner, "Foundation and Practice of Neurofeedback for the Treatment of Epilepsy", Applied Psychophysiology \& Biofeedback, March 2006.

[12] James R. Evans, Andrew Barbanel Aptos, Introduction to Quantitative EEG and neurofeedback, California academic press, an imprint of Elsevier,2000.

[13] Qiang Wang, Olga Sourina, and Minh Khoa Nguyen, "EEG-based "Serious" Games Design for Medical Applications", IEEE International Conference on Cyberworlds, 2010.

[14] Attwood, H. L., and MacKay, W. A., Essentials of Neurophysiology, B. C. Decker, Hamilton, Canada, 1989.

[15] Nunez, P. L., Neocortical Dynamics and Human EEG Rhythms, Oxford University Press, New York, 1995.

[16] Teplan, M., 'Fundamentals of EEG measurements', Measmt Sci. Rev., 2(2), 2002.

[17] Bickford, R. D., 'Electroencephalography', in Encyclopedia of Neuroscience, Ed. G. Adelman, Birkhauser, Cambridge (USA), 1987, 371-373.

[18] Saeid Sanei and J. A. Chambers, "EEG SIGNAL PROCESSING”, John Wiley \& Sons Ltd., 2007.

[19] Sterman, M. B., MacDonald, L. R., and Stone, R. K., 'Biofeedback training of sensorimotor EEG in man and its effect on epilepsy', Epilepsia, 15, 1974, 395-416.

[20] Ashwal, S., and Rust, R., 'Child neurology in the 20th century', Pedia. Res., 53, 2003, 345-361.

[21] Niedermeyer, E., 'The normal EEG of the waking adult', Chapter 10, in Electroencephalography, Basic Principles, Clinical Applications, and Related Fields, Eds E. Niedermeyer and F. Lopes da Silva, 4th edn, Lippincott, Williams and Wilkins, Philadelphia, Pennsylvania, 1999, 174-188.

[22] Pfurtscheller, G., Flotzinger, D., and Neuper, C., 'Differentiation between finger, toe and tongue movement in man based on $40 \mathrm{~Hz}$ EEG', Electroencephalogr. Clin. Neurophysiol., 90, 1994, 456460.

[23] Wanda Wyrwicka, Maurice B. Sterman, "Instrumental Conditioning of Sensorimotor Cortex EEG Spindles in the Waking Cat" Physiology and Behavior. Vol 3, pp 703-707 Pergamon Press, 1968

[24] Jasper, H., 'Report of committee on methods of clinical exam in EEG', Electroencephalogr. Clin. Neurophysiol., $10,1958,370-375$.

[25] Nayak, D., Valentin, A., Alarcon, G., Seoane, J. J. G., Brunnhuber, F., Juler, J., Polkey, C. E., and Binnie, C. D., 'Characteristics of scalp electrical fields associated with deep medial temporal epileptiform discharges', Clin. Neurophysiol., 115, 2004, 1423-1435.

[26] Barrett, G., Blumhardt, L., Halliday, L., Halliday, A. M., and Kriss, A., 'A paradox in the lateralization of the visual evoked responses', Nature, 261, 1976, 253-255.

[27] Halliday, A. M., Evoked potentials in neurological disorders', in Event-Related Brain Potentials in Man, Eds E. Calloway, P. Tueting, and S. H. Coslow, Academic Press, New York, 1978, 197-210. 
[28] Cherry, C. E., 'Some experiments in the recognition of speech, with one and two ears', J. Acoust. Soc. Am., 25, 1953, 975-979.

[29] Herault, J., and Jutten, C., 'Space or time adaptive signal processing by neural models', Proceeding of the American Institute of Physics (AIP) Conference on Neural Networks for Computing, 1986 pp. 206-211.

[30] P. F"oldi'ak, and Young, M., 'Sparse coding in the primate cortex', in The Handbook of Brain Theory and Neural Networks, Eds Michael A. Arbib, 2nd edn., MIT Press, Cambridge, Massachussetts, 1995, pp. 895-898.
[31] Einhauser, W., Kayser, C., Konig, P., and Kording, K. P., 'Learning the invariance properties of complex cells from their responses to natural stimuli', Eur. J. Neurosci., $15,2002,475-486$.

[32] Jutten, C., and Karhunen, J., 'Advances in blind source separation (BSS) and independent component analysis (ICA) for nonlinear mixtures', Int. J. Neural Systems, 14(5), 2004, 267-292.

[33] Almeida, L. B., 'Nonlinear Source Separation', in Synthesis Lectures on Signal Processing, Ed. J. Moura, Morgan \& Claypool, California, USA, 2006 